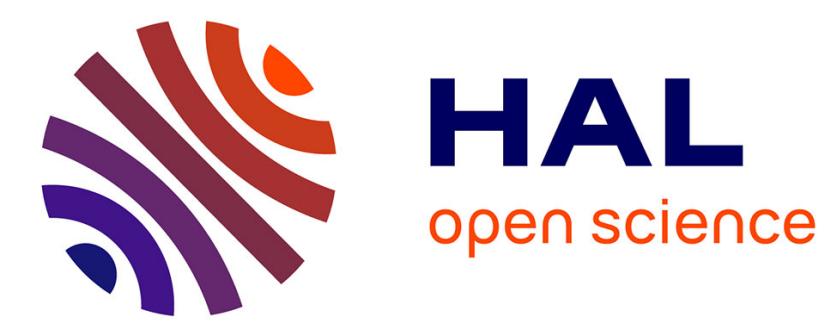

\title{
Ions in solutions: Determining their polarizabilities from first-principles
}

\author{
J.J. Molina, S. Lectez, S. Tazi, M. Salanne, Jean-François Dufrêche, J. \\ Roques, E. Simoni, P. A. Madden, P. Turq
}

\section{- To cite this version:}

J.J. Molina, S. Lectez, S. Tazi, M. Salanne, Jean-François Dufrêche, et al.. Ions in solutions: Determining their polarizabilities from first-principles. Journal of Chemical Physics, 2011, 134, pp.014511. 10.1063/1.3518101 . in2p3-00575117

\section{HAL Id: in2p3-00575117 https://hal.in2p3.fr/in2p3-00575117}

Submitted on 9 Jun 2021

HAL is a multi-disciplinary open access archive for the deposit and dissemination of scientific research documents, whether they are published or not. The documents may come from teaching and research institutions in France or abroad, or from public or private research centers.
L'archive ouverte pluridisciplinaire HAL, est destinée au dépôt et à la diffusion de documents scientifiques de niveau recherche, publiés ou non, émanant des établissements d'enseignement et de recherche français ou étrangers, des laboratoires publics ou privés. 


\title{
lons in solutions: Determining their polarizabilities from first-principles
}

\author{
John J. Molina, ${ }^{1,2,3}$ Sébastien Lectez, ${ }^{4}$ Sami Tazi, ${ }^{1,2}$ Mathieu Salanne, ${ }^{1,2, a)}$ Jean-François \\ Dufrêche ${ }^{3}$ Jérôme Roques, ${ }^{4}$ Eric Simoni, ${ }^{4}$ Paul A. Madden, ${ }^{5}$ and Pierre Turq ${ }^{1,2,3}$ \\ ${ }^{1}$ UPMC Univ Paris 06, UMR 7195, PECSA, F-75005, Paris, France \\ ${ }^{2}$ CNRS, UMR 7195, PECSA, F-75005, Paris, France \\ ${ }^{3}$ Institut de Chimie Séparative de Marcoule (ICSM), UMR 5257, Université Montpellier 2, CEA, CNRS, Site de \\ Marcoule, Bâtiment 426, BP 17171, F-30200, Bagnols-sur-Cèze, France \\ ${ }^{4}$ Institut de Physique Nuclaire d'Orsay, Université Paris-Sud 11, CNRS UMR 8608, 15 rue Georges \\ Clémenceau, Bâtiment 100, F-91406, ORSAY Cedex, France \\ ${ }^{5}$ Department of Materials, University of Oxford, Parks Road, Oxford OX1 3PH, United Kingdom
}

(Received 30 September 2010; accepted 29 October 2010; published online 7 January 2011)

Dipole polarizabilities of a series of ions in aqueous solutions are computed from first-principles. The procedure is based on the study of the linear response of the maximally localized Wannier functions to an applied external field, within density functional theory. For most monoatomic cations $\left(\mathrm{Li}^{+}\right.$, $\mathrm{Na}^{+}, \mathrm{K}^{+}, \mathrm{Rb}^{+}, \mathrm{Mg}^{2+}, \mathrm{Ca}^{2+}$ and $\mathrm{Sr}^{2+}$ ) the computed polarizabilities are the same as in the gas phase. For $\mathrm{Cs}^{+}$and a series of anions $\left(\mathrm{F}^{-}, \mathrm{Cl}^{-}, \mathrm{Br}^{-}\right.$and $\left.\mathrm{I}^{-}\right)$, environmental effects are observed, which reduce the polarizabilities in aqueous solutions with respect to their gas phase values. The polarizabilities of $\mathrm{H}_{(\mathrm{aq})}^{+}, \mathrm{OH}_{(\mathrm{aq})}^{-}$have also been determined along an ab initio molecular dynamics simulation. We observe that the polarizability of a molecule instantaneously switches upon proton transfer events. Finally, we also computed the polarizability tensor in the case of a strongly anisotropic molecular ion, $\mathrm{UO}_{2}^{2+}$. The results of these calculations will be useful in building interaction potentials that include polarization effects. (C) 2011 American Institute of Physics. [doi:10.1063/1.3518101]

\section{INTRODUCTION}

Small inorganic ions play a central role in many fields of chemistry. In biological systems for example, not only do they influence the solvation of proteins, ${ }^{1}$ but they also play a fundamental role in the protein's diffusion mechanism along DNA. ${ }^{2}$ In environmental science, the hydrophobicity of ions has been shown to be the driving force behind their affinity for clay systems, which will have important consequences in the evaluation of these materials for nuclear waste storage applications. ${ }^{3}$

Probably due to their apparent simplicity, ionic solutions have long been studied by molecular dynamics (MD) simulations employing somewhat crude models, which only take into account the electrostatic, van der Waals repulsion, and dispersion interactions. The induction term, which is recognized as providing an important contribution to the interaction energy in condensed phase systems, was accounted for in an average way; for example, by enhancing the dipole moment of the water molecule compared to its gas phase value. ${ }^{4-6}$ After several important yet isolated attempts, it was only recently that simulations involving an explicit induction term were systematically performed for ionic solutions. ${ }^{7-9}$ In such models [polarizable models (PMs)], the induction effects are most often introduced through a self-consistent calculation of the induced dipole moments of each ion or molecule (although some Car-Parrinello type implementations have also been employed). They are therefore accurate for

a) Author to whom correspondence should be addressed. Electronic mail: mathieu.salanne@upmc.fr. systems in which higher-order induced multipole moments remain small. For example, this approximation will hold when describing alkali ions, but not for ions with high quadrupole polarizabilities like $\mathrm{Ag}^{+} .10,11$

The reason for the success of the PMs is their enhanced transferability. They provide a better description of ionic solutions at interfaces and in the vicinity of complex organic molecules such as proteins, i.e., in systems for which the inclusion of induction, even in an approximate way, is sufficent to obtain the correct trends for physico-chemical properties. ${ }^{12-15}$ However, their use for determining precise quantities, such as solvation free energies in bulk water, has been somewhat hindered due to their rough parameterization, which was mainly done by adding a polarization term to existing classical force fields. It is only recently that a systematic parameterization of PMs for aqueous systems was proposed, in the framework of the classical Drude oscillators. ${ }^{16}$ Such potentials have been developed for purely ionic systems for many years. ${ }^{17}$

Among the series of parameters that have to be determined during the building of a PM, one has an evident physical meaning: The electronic polarizability tensor of the ion, which measures the relative tendency of its electron cloud to be distorted from its normal shape by an electric field. Although one could think that the polarizability of a molecule or an ion is an intrinsic property, which remains unchanged from the gas phase to any condensed phase, this is not the case. This is particularly true in the case of anions because they often have much larger polarizabilities than cations. For example, the polarizability of the oxide anion switches from 1.3-1.4 $\AA^{3}$ in $\mathrm{B}_{2} \mathrm{O}_{3}, \mathrm{Al}_{2} \mathrm{O}_{3}$ and $\mathrm{SiO}_{2}$ to $3.6 \AA^{3}$ in $\mathrm{BaO} .{ }^{18}$ In their param- 
eterization of PMs, Yu et al. ${ }^{16}$ have therefore scaled down the gas phase polarizabilities of the anions by a factor of 0.724 , while keeping the same values for cations. In this work we propose a direct computation of the polarizability for a series of simple inorganic ions in solution, namely $\mathrm{F}^{-}, \mathrm{Cl}^{-}, \mathrm{Br}^{-}, \mathrm{I}^{-}$, $\mathrm{OH}_{(\mathrm{aq})}^{-}, \mathrm{Na}^{+}, \mathrm{K}^{+}, \mathrm{Rb}^{+}, \mathrm{Cs}^{+}, \mathrm{H}_{(\mathrm{aq})}^{+}, \mathrm{Mg}^{2+}, \mathrm{Sr}^{2+}$, and $\mathrm{Ca}^{2+}$. We have also studied the case of a more complicated, triatomic cation, the uranyl $\mathrm{UO}_{2}^{2+}$. Our method for determining condensed phase polarizabilities involves density functional theory (DFT) calculations; it is based on the linear response of the Wannier orbitals to an external electric field, ${ }^{19-21}$ as explained in Sec. II.

\section{NUMERICAL METHODS}

\section{A. Polarizabilities from DFT}

DFT calculations on periodic systems are based on the Kohn-Sham (KS) method to determine the ground state electronic wavefunction of a given ionic configuration. One of its drawbacks is the delocalization of the electronic density throughout the whole simulation cell, which prevents any straightforward extraction of the individual electronic properties of a given atom or molecule.

The use of the maximally localized Wannier function (MLWF) formalism ${ }^{22}$ is an efficient way to overcome this difficulty. The MLWFs provide a picture of the electron distribution around atoms which is easily interpreted from a chemical point of view. They are determined by unitary transformations of the KS eigenvectors

$$
\left|\phi_{n}^{w}\right\rangle=\sum_{m=1}^{N} U_{n m}\left|\phi_{m}\right\rangle
$$

where the sum runs over all the occupied KS states $\left\{\phi_{n}\right\}_{n \in[1, N]}$, and the unitary matrix $\boldsymbol{U}$ is determined by iterative minimization of the Wannier function spread $\Omega$, which is defined as

$$
\Omega=-\frac{1}{(2 \pi)^{2}} \sum_{n=1}^{N} \log \left|s_{n}\right|^{2} ; \quad s_{n, \alpha}=\left\langle\phi_{n}^{w}\left|e^{i(2 \pi / L) r_{\alpha}}\right| \phi_{n}^{w}\right\rangle
$$

when periodic boundary conditions are applied, where $\alpha$ refers to the coordinates axis, $x, y$, and $z$.

A complete theory of electric polarization in crystalline dielectrics has been developed in recent years, ${ }^{23-25}$ which validates the calculation of the dipole moments of single ions or molecules from the center of charge of the subset of MLWF that are localized in their vicinity. ${ }^{26-28}$ The MLWF centers are computed according to ${ }^{26}$

$$
r_{n, \alpha}^{w}=-\frac{L}{2 \pi} \Im\left(\log s_{n, \alpha}\right)
$$

and the partial dipole moment of a given ion or molecule $I$ is defined, in atomic units, as

$$
\boldsymbol{\mu}^{I}=\sum_{i \in I}\left(Z_{i} \boldsymbol{R}_{i}-2 \sum_{n \in i} \boldsymbol{r}_{n}^{w}\right)
$$

where the sum over $i \in I$ includes all atoms belonging to $I$, with $Z_{i}$ and $\boldsymbol{R}_{i}$ their nuclear charge and position, and the sum over $n \in i$ includes all the MLWF whose center is localized in the vicinity of $\boldsymbol{R}_{i}$. When a small external electric field $\mathcal{E}$ is applied to the system, the linear response may be characterized by an additional field-induced dipole moment $\delta \boldsymbol{\mu}^{I}$ on each individual molecule. It is convenient to think of the applied field as an optical field, in order to distinguish its effect from that of the static fields, which are caused by the permanent charge distributions of the molecules, and to think of $\delta \boldsymbol{\mu}^{I}$ as the net induced dipole that is oscillating at the optical frequency. For an electronically insulating material, the induced dipole can be written in terms of the total (optical frequency) electric field which acts on it

$$
\delta \boldsymbol{\mu}^{I}\left(\left\{\boldsymbol{R}^{N}\right\}\right)=\boldsymbol{\alpha}^{I}\left(\left\{\boldsymbol{R}^{N}\right\}\right) \cdot\left[\mathcal{E}+\sum_{J \neq I} \hat{\boldsymbol{T}}^{I J} \cdot \delta \boldsymbol{\mu}^{J}\left(\left\{\boldsymbol{R}^{N}\right\}\right)\right]
$$

where the sum runs over all polarizable entities (ions or molecules) $J \neq I$ in the system. In this equation, we have introduced the dipole polarizability tensor $\boldsymbol{\alpha}^{I}\left(\left\{\boldsymbol{R}^{N}\right\}\right)$ of molecule $I$, for the particular condensed phase configuration $\left\{\boldsymbol{R}^{N}\right\}$. Also included is the dipole-dipole interaction tensor, $\hat{\boldsymbol{T}}^{I J}$, whose components are defined by $\hat{T}_{\alpha \beta}^{I J}=\nabla_{\alpha} \nabla_{\beta} 1 / \hat{r}^{I J}$; in practice, for a periodic system, it will be computed using the Ewald summation technique. ${ }^{29,30}$ The first term on the right-hand side of the equation represents the direct contribution of the external electric field to the induced dipole; the second-term is the contribution of the reradiated electric fields due to the dipoles that are induced in all the other molecules $(J \neq I)$ in the sample. In principle, higher-order induced multipoles also contribute to this expansion, but we will ignore them. In a uniform external field, the directly induced higherorder multipoles on spherical atoms and ions vanish, and even for molecules, their effect is expected to be much smaller than that of the dipoles.

In DFT calculations on periodic systems, the coupling between the external electric field and the electronic system is expressed through the macroscopic polarization of the periodically replicated cell ${ }^{31,32}$ and is defined using the Berry phase approach of Resta. ${ }^{33}$ It is then possible to determine the new partial dipole moment for each species in the presence of a field, via another localization step. The field induced dipoles are calculated from the difference between the total molecular dipoles in the presence and absence of the field.

Equation (5) can be inverted to determine the individual electronic polarizabilities for that particular condensedphase configuration $\left\{\boldsymbol{R}^{N}\right\}$. Consider the application of fields $\mathcal{E}^{(\alpha)}$, along each Cartesian direction $\alpha=x, y, z$, and denote by $\left\{\delta \boldsymbol{\mu}^{I,(\alpha)}\right\}_{I \in[1, N]}$ the corresponding values of the induced dipole moments. The total field $\boldsymbol{f}^{I,(\alpha)}$ at each position $\boldsymbol{R}_{I}$ can be obtained from

$$
\boldsymbol{f}^{I,(\alpha)}=\mathcal{E}^{(\alpha)}+\sum_{J \neq I} \hat{\boldsymbol{T}}^{I J} \cdot \boldsymbol{\delta} \boldsymbol{\mu}^{J,(\alpha)},
$$

which is conveniently evaluated from the electric field given by a dipolar Ewald sum. Finally, the polarizability tensor of molecule $I$ is given by

$$
\boldsymbol{\alpha}^{I}\left(\left\{\boldsymbol{R}^{N}\right\}\right)=\left(\boldsymbol{F}^{I}\right)^{-1} \cdot \boldsymbol{\Pi}^{I},
$$


where $\boldsymbol{F}^{I}$ and $\boldsymbol{\Pi}^{I}$ are second-rank three-dimensional tensors defined as

$$
\begin{aligned}
& F_{\alpha \beta}^{I}=f_{\alpha}^{I,(\beta)}, \\
& \Pi_{\alpha \beta}^{I}=\delta \mu_{\alpha}^{I,(\beta)} .
\end{aligned}
$$

\section{B. Computation details}

The polarizabilities were calculated as described above using the CPMD package. ${ }^{34}$ For calculations with an applied external field, its intensity was set to a value of 0.001 a.u. The gradient-corrected BLYP (in the case of $\mathrm{Rb}^{+}$a PBE functional was used instead ${ }^{35}$ ) functional $^{36,37}$ was used in conjunction with Troullier-Martins ${ }^{38}\left(\mathrm{Cl}^{-}, \mathrm{Br}^{-}, \mathrm{Cs}^{+}\right.$, and $\mathrm{K}^{+}$) and Goedecker-Teter-Hutter ${ }^{39-41}$ (GTH) pseudopotentials $\left(\mathrm{I}^{-}, \mathrm{Na}^{+}, \mathrm{Rb}^{+}, \mathrm{Mg}^{2+}, \mathrm{Ca}^{2+}, \mathrm{Sr}^{2+}\right.$ ), and a plane-wave basis set with an energy cutoff of at least 70 Ryd. A minimum of 100 configurations were analyzed for each ionic species. In a recent study, various density functionals were benchmarked against calculations at the coupled-cluster level of theory, for the dipole polarizabilities of water clusters $n=2-12 .^{42}$ It was shown that the hybrid functionals provide more accurate results. Unfortunately, the use of such functionals for the condensed-phase systems considered here would entail computational costs too high to be of practical use. Errors of a few percents, therefore, have to be expected in our calculated values.

The configurations were extracted from classical molecular dynamics simulations at a temperature of $T=300 \mathrm{~K}$. A periodically replicated cubic simulation box, with one ion and at least 31 water molecules, was used. The monovalent ions $\mathrm{F}^{-}, \mathrm{Cl}^{-}, \mathrm{Br}^{-}, \mathrm{I}^{-}, \mathrm{Li}^{-}, \mathrm{Na}^{-}, \mathrm{K}^{-}, \mathrm{Rb}^{-}$, and $\mathrm{Cs}^{-}$were described using the nonpolarizable potentials of Dang et al., ${ }^{8,43,44}$ while the polarizable potentials of $\mathrm{Yu}$ and coworkers ${ }^{16}$ were used for the divalent ions $\mathrm{Mg}^{2+}, \mathrm{Ca}^{2+}$, and $\mathrm{Sr}^{2+}$. For the series of monovalent ions, the water molecules were represented using the nonpolarizable $\mathrm{SPC} / \mathrm{E}^{45}$ model whereas the polarizable model from Dang and Chang ${ }^{46}$ was used in the case of the divalent ions.

In the case of $\mathrm{H}_{(\mathrm{aq})}^{+}$and $\mathrm{OH}_{(\mathrm{aq})}^{-}$, the configurations were extracted from Born-Oppenheimer molecular dynamics simulations ${ }^{47}$ performed using the CP2K package. ${ }^{48}$ Here also, we used the BLYP functional, ${ }^{36,37}$ with GTH pseudopotentials. A 280 Ryd plane wave density cutoff has been applied and the molecularly optimized (MOLOPT) Gaussian basis sets were employed. ${ }^{49}$ The cubic simulation cell, with a lateral size of $9.865 \AA$, initially contained $31 \mathrm{H}_{2} \mathrm{O}$ molecules and one $\mathrm{H}_{3} \mathrm{O}^{+}\left(\mathrm{OH}^{-}\right)$ion. The time step for the MD simulation was $0.5 \mathrm{fs}$, for a total simulations time of $10 \mathrm{ps}\left(\mathrm{H}_{(\mathrm{aq})}^{+}\right)$ and $20 \mathrm{ps}\left(\mathrm{OH}_{(\mathrm{aq})}^{-}\right)$, respectively (after 2 ps of equilibration in both cases). NVT conditions were imposed by a NoseHoover thermostat with a target temperature of $350 \mathrm{~K}$.

Finally, for $\mathrm{UO}_{2}^{2+}$, the configurations were extracted from a Car-Parrinello molecular dynamics simulation ${ }^{50}$ performed using the CPMD package. The PBE functional ${ }^{35}$ was used with Troullier-Martins pseudopotentials. ${ }^{38}$ A 80 Ryd energy cutoff had been applied. The cubic simulation cell, with a

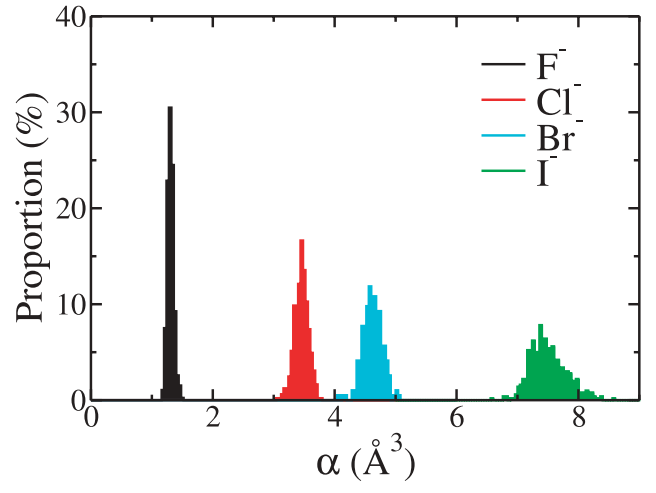

FIG. 1. Distribution of the isotropic polarizabilities $\bar{\alpha}=\frac{1}{3} \operatorname{Tr}(\boldsymbol{\alpha})$ of halide ions in liquid water.

lateral size of $12.41 \AA$, contained $61 \mathrm{H}_{2} \mathrm{O}$ molecules and one $\mathrm{UO}_{2}^{2+}$ ion. The fictitious electron mass was set to 600 a.u. and the time step was $0.121 \mathrm{fs}$, for a total simulation time of $8.4 \mathrm{ps}$, after 2 ps of equilibration. NVT conditions were imposed by a Nose-Hoover thermostat with a target temperature of $350 \mathrm{~K}$.

\section{RESULTS}

\section{A. Monoatomic ions}

For all monoatomic ions, the polarizability tensor has offdiagonal components equal to 0 , and is on average isotropic. An example of distributions for $\bar{\alpha}=\frac{1}{3} \operatorname{Tr}(\boldsymbol{\alpha})$ is given for the series of halide ions in Fig. 1. From these distributions, we can determine the average isotropic polarizability of each species. These are reported in Table I.

The only previous attempt to calculate such polarizabilities for ions in solution was focused solely on the nitrate, ${ }^{51}$ sulfate $^{52}$ and chloride ${ }^{53}$ anions. We can therefore compare our data to the latter only. In their study, Jungwirth and Tobias ${ }^{53}$ calculated the electrostatic contribution to the confining potential felt by the ions, using a point charge representation for the static charge distribution of solvating water molecules. In

TABLE I. Average values of the isotropic polarizability $\langle\bar{\alpha}\rangle$ of monoatomic ions. All values are in $\AA^{3}$. Also reported are the values employed in common force fields and the gas-phase polarizabilities (Refs. 56 and 57).

\begin{tabular}{lccccc}
\hline \hline Ion & This work & SWM4-NDP $^{\mathrm{a}}$ & Amoeba $^{\mathrm{b}}$ & DC97 & Gas phase \\
\hline $\mathrm{F}^{-}$ & 1.3 & 1.786 & 1.350 & 1.050 & 2.467 \\
$\mathrm{Cl}^{-}$ & 3.5 & 3.969 & 4.000 & 3.690 & 5.482 \\
$\mathrm{Br}^{-}$ & 4.6 & 5.262 & 5.650 & 4.770 & 7.268 \\
$\mathrm{I}^{-}$ & 7.5 & 7.439 & 7.250 & 6.920 & 10.275 \\
$\mathrm{Li}^{+}$ & 0.029 & 0.032 & 0.028 & 0.029 & 0.032 \\
$\mathrm{Na}^{+}$ & 0.18 & 0.157 & 0.120 & 0.240 & 0.157 \\
$\mathrm{~K}^{+}$ & 0.81 & 0.830 & 0.780 & 0.830 & 0.830 \\
$\mathrm{Rb}^{+}$ & 1.32 & 1.370 & 1.350 & - & 1.370 \\
$\mathrm{Cs}^{+}$ & 2.02 & 2.360 & 2.260 & 2.440 & 2.360 \\
$\mathrm{Mg}^{2+}$ & 0.08 & 0.075 & $0.080(0.107)$ & - & 0.075 \\
$\mathrm{Ca}^{2+}$ & 0.44 & 0.490 & $0.550(0.376)$ & - & 0.490 \\
$\mathrm{Sr}^{2+}$ & 0.81 & 0.870 & - & - & 0.870 \\
\hline \hline
\end{tabular}

${ }^{a}$ Reference 16

${ }^{\mathrm{b}}$ Provided in the TINKER molecular modeling simulation package (Ref. 66) for Amoeba (Refs. 62-64) except values in parenthesis, which are from Ref. 65.

${ }^{\mathrm{c}}$ From DC97 (Refs. 7, 12, 46, and 67) force fields. 
a first attempt, they used theSPC/E charge model, and they obtained polarizabilities of around $4.2 \AA^{3}$. They then used the information extracted from the Wannier localization procedure to determine the water charge distribution. In that case, net charges of $+6,+1$, and -2 were respectively placed on the positions of oxygen atoms, hydrogen atoms, and Wannier centers, ${ }^{26,54}$ and a smaller polarizability of $3.9 \AA^{3}$ was obtained. This remains higher than our proposed value $\left(3.5 \AA^{3}\right)$ for several reasons. First, due to the lack of statistics, since only five snapshots were used. Second, the partial charge model may be too simple; in which case, higher-order moments of the Wannier functions should be used to improve the representation of the electrostatic potential. ${ }^{55}$ Finally, in this calculation the multipole-induced dipole contributions are not taken into account.

The gas phase isotropic polarizabilities ${ }^{56,57}$ also reported in Table I, enable us to quantify the importance of environmental effects on the polarizabilities of ions in solution. These effects are well established for the case of anions in ionic crystals or liquids. ${ }^{58,59}$ They are due to the existence of a confining potential, which affects the electron density around a given species, and originates from both Coulombic interactions and the exclusion of electrons from the region occupied by the electron density of the first-neighbor solvation shell. ${ }^{60}$ Here we observe the most important effects for anions: the polarizability of the fluoride anion is reduced by as much as $47 \%$ with respect to its gas value in solution. This effect becomes less pronounced for species with bigger radii, with the polarizability of the iodide anion only being reduced by $27 \%$. In the case of cations, almost no effect is observed for any of them, except $\mathrm{Cs}^{+}$, which is by far the most polarizable one. In Table I we also provide the atomic polarizabilities employed in common force fields. These values, which were obtained empirically ${ }^{61}$ or by fitting some ab inito data, ${ }^{62-65}$ correctly reproduce the environmental effects, although some discrepancies are observed, particularly in the case of $\mathrm{F}^{-}$and $\mathrm{Br}^{-}$.

In a recent work, Bauer et al. ${ }^{68}$ studied the variation of $\mathrm{F}^{-}, \mathrm{Cl}^{-}$, and $\mathrm{Br}^{-}$ions polarizability in water clusters with respect to the corresponding gas phase values. Their method is based on a Hirshfeld ${ }^{69}$ partitioning of the electronic density, which is obtained by ab initio calculations at the B3LYP level of theory. The strong basis set dependence they have observed, as well as the system size limitation, and the fact that the induced dipole-induced dipole contributions are not explicitly taken into account, makes it difficult to establish a clear comparison with the values given here for bulk systems, but their results are in good agreement with the present study, showing a significant reduction of ion polarizability of halide anions upon solvation by water molecules.

\section{B. Ionic product of liquid water}

The same procedure has been applied to two solutions of liquid water respectively containing one $\mathrm{H}_{(\mathrm{aq})}^{+}$and one $\mathrm{OH}_{(\mathrm{aq})}^{-}$. Despite the importance of these two species, it is only recently that the chemical nature and transport properties have been determined by molecular dynamics simulations. ${ }^{70-76} \mathrm{In}$

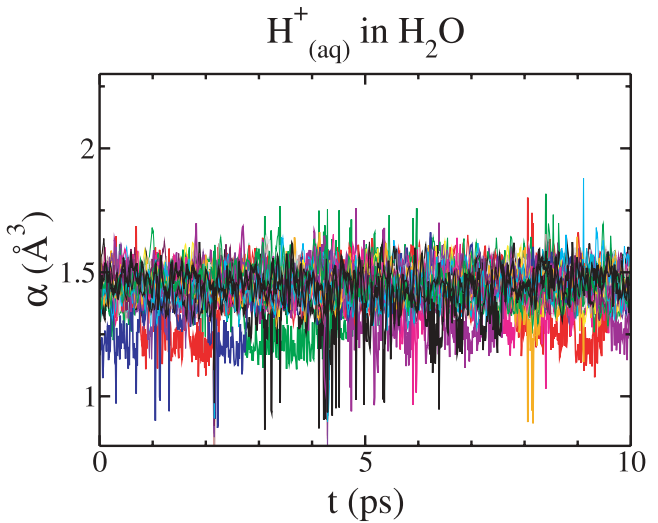

(a)

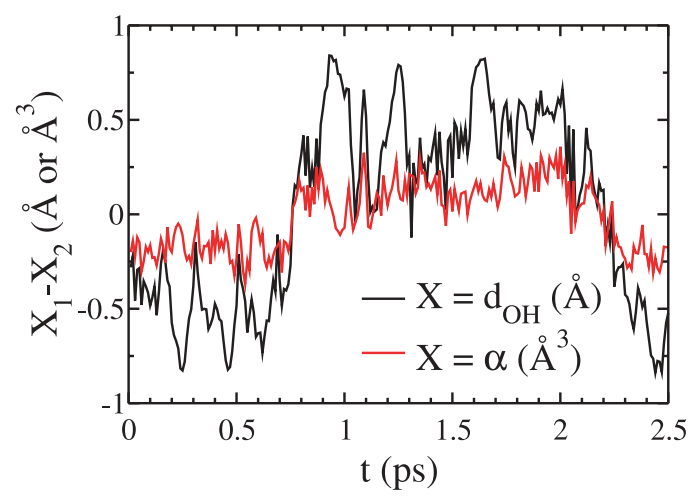

(b)

FIG. 2. Top: Isotropic polarizabilities of all molecules during an $a b$ initio molecular dynamics (AIMD) simulation of a system consisting of $1 \mathrm{H}^{+}+32$ $\mathrm{H}_{2} \mathrm{O}$. Bottom: Difference between the O-H distances $\Delta d$ and the molecular polarizabilities $\Delta \alpha$ of the two molecules involved in a proton transfer event.

the top panel of Fig. 2, we show the time evolution of the isotropic polarizability for all the molecules in the simulation box containing $\mathrm{H}_{(\text {aq) }}^{+}$. We observe that there is always exactly one molecule that has a polarizability that differs from the others, although the index of this molecule changes during the simulation. This is due to the transfer of protons through the Grotthus mechanism. ${ }^{70-72}$ As for monoatomic ions, the polarizability tensor of the $\mathrm{H}_{3} \mathrm{O}^{+}$ion is almost isotropic and we obtain an average isotropic polarizability of $1.19 \AA^{3}$. In the bottom panel of Fig. 2, we focus on a proton exchange involving two molecules: First, the proton is bonded to the oxygen atom labeled 1, then it jumps on the nearest oxygen atom, labeled 2, during $\approx 1.3 \mathrm{ps}$, before coming back to its original position. In Fig. 2, the exchange is observed using the usual quantity, ${ }^{71,72}$ $\Delta d=d_{\mathrm{O}_{1} \mathrm{H}}-d_{\mathrm{O}_{2} \mathrm{H}}$ : Proton transfer occurs when this function equals 0 . We also calculated the polarizability difference between the two oxygen atoms, $\Delta \alpha=\alpha_{1}-\alpha_{2}$, and observed that this function follows the same variations as $\Delta d$. This means that the polarizability of the molecule instantaneously follows its chemical nature when a chemical reaction occurs.

The computed value of $\alpha$ differs from the one employed in a series of recent molecular dynamics simulation of $\mathrm{H}_{3} \mathrm{O}^{+}$ in water using $\mathrm{PM},{ }^{77,78}$ which was of $0.98 \AA^{3}$. It should be noted, however, that in the same studies some simulations with the $\mathrm{H}_{5} \mathrm{O}_{2}^{+}$ion were also performed, for which polarizabilities of $1.19 \AA^{3}$ were attributed to both oxygen atoms. 


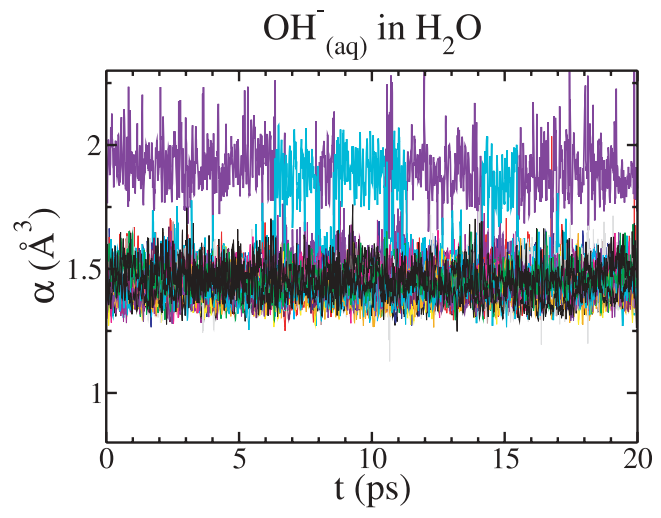

FIG. 3. Isotropic polarizabilities of all molecules during an AIMD simulation of a system consisting of $1 \mathrm{OH}^{-}+31 \mathrm{H}_{2} \mathrm{O}$.

Our value is in good agreement with the results of Buin and Iftimie, who computed condensed-phase polarizabilities in the acidic $\mathrm{H}_{2} \mathrm{O}-\mathrm{HF}$ mixtures. ${ }^{79}$

In the case of the hydroxide anion simulation, a similar behavior is observed for the time variation of the isotropic polarizabilities (Fig. 3). Considerably less transfer events are observed than in the case of $\mathrm{H}_{(\mathrm{aq})}^{+}$, during the same period of time, in good agreement with previous studies comparing these two systems. The average isotropic polarizability obtained for the $\mathrm{OH}^{-}$ion is of $1.91 \AA^{3}$, a value slightly smaller than the one used in previous classical MD simulations. ${ }^{80}$ The increase of the polarizability when passing from $\mathrm{H}_{3} \mathrm{O}^{+}$ to $\mathrm{H}_{2} \mathrm{O}$, and then to $\mathrm{OH}^{-}$, shows that the formation of bonds between the oxygen and hydrogen atoms provokes a compression of the electronic cloud of the molecule. When switching to the molecular coordinate system (where the $x$ axis is along the $\mathrm{O}-\mathrm{H}$ bond), we observe a slight anisotropy, and the diagonal components are $\alpha_{x x}=1.84 \AA^{3}, \alpha_{y y}=\alpha_{z z}=1.95 \AA^{3}$. The difference between the components is small, which justifies the use of isotropic polarizabilities in molecular dynamics simulations involving the hydroxide anion.

\section{An example of an anisotropic molecular ion: The uranyl $\mathrm{UO}_{2}^{2+}$}

The uranyl cation is a triatomic linear molecule. In water, its first solvation shell consists of five water molecules surrounding the uranium atom. ${ }^{81,82}$ This example is particularly interesting, since the electron cloud shape should be very anisotropic. The oxygen atoms, which are very electronegative, should attract most of the electron density. Our results confirm this picture. First, the $\mathrm{UO}_{2}^{2+}$ is by far more polarizable than all the other ions studied in this work, with an average molecular polarizability of $4.87 \AA^{3}$. Second, in the molecular coordinate system (where the $x$ axis is along the U-O bonds), we observe a strong anisotropy: The diagonal components are $\alpha_{x x}=10.55 \AA^{3}, \alpha_{y y}=\alpha_{z z}=2.07 \AA^{3}$. This means that it is very important to take induction effects into account when performing classical molecular dynamics simulations involving this cation. It is all the more important since the uranium atom is at an oxydation degree of $+\mathrm{VI}$, which implies that the surrounding water molecules, within the first solvation shell, will experience a strong electric field and thus an enhanced dipole moment.

\section{CONCLUSION}

We have shown how to obtain dipole polarizabilites of ions in condensed phases, within density functional theory, by measuring the linear response of the MLWF to an externally applied electric field. The calculated values for the isotropic polarizabilites of $\mathrm{F}^{-}, \mathrm{Cl}^{-}, \mathrm{Br}^{-}, \mathrm{I}^{-}, \mathrm{OH}_{(\mathrm{aq})}^{-}, \mathrm{Na}^{+}, \mathrm{K}^{+}, \mathrm{Rb}^{+}$, $\mathrm{Cs}^{+}, \mathrm{H}_{\text {(aq) }}^{+}, \mathrm{Mg}^{2+}, \mathrm{Sr}^{2+}, \mathrm{Ca}^{2+}$, and $\mathrm{UO}_{2}^{2+}$ in aqueous solution, were 1.3, 3.5, 4.6, 7.5, 1.91, 0.18, 0.81, 1.32, 2.02, 1.19, 0.08, $0.44,0.81$, and $4.87 \AA^{3}$, respectively. In the case of $\mathrm{UO}_{2}^{2+}$ the polarizability tensor is strongly anisotropic $\left(\alpha_{x x}=10.55 \AA^{3}\right.$, $\alpha_{y y}=\alpha_{z z}=2.07 \AA^{3}$ ). A noticeable difference is observed between the behavior of polarizabilities of cations and anions, with the latter showing a much stronger environmental dependence. On average the condensed-phase values of anion polarizabilites are found to be 50 to $70 \%$ of their gas phase values, with the effect being smaller for larger ions. In the case of cations, the variation is considerably less important; the largest difference was observed for $\mathrm{Cs}^{+}$, but its polarizability is only reduced by $15 \%$. This is all the more important, since for many systems the anions experience a more disordered environment than the cations. It is crucial, therefore, to use accurate polarizabilities for the anions in molecular dynamics simulations. The present calculations will provide useful input for the systematic development of polarizable models. The inclusion of induction effects will also require the determination of short-range damping effects, which are very important in the case of ionic systems. ${ }^{15,17,21}$ The corresponding parameters can rather easily be determined from a dipolefitting procedure. ${ }^{83}$

\section{ACKNOWLEDGMENTS}

The authors acknowledge the support of the French Agence Nationale de la Recherche (ANR), under GrantSYSCOMM (ANR-09-SYSC-012) "Multiscale Simulation of Ions at Solid-Liquid Interfaces," and of the CNRS under the GNR PARIS (PACEN program).

${ }^{1}$ J. Heyda, J. C. Vincent, D. J. Tobias, J. Dzubiella, and P. Jungwirth, J. Phys. Chem. B 114, 1213 (2010).

${ }^{2}$ V. Dahirel, F. Paillusson, M. Jardat, M. Barbi, and J. M. Victor, Phys. Rev. Lett. 102, 228101 (2009).

${ }^{3}$ B. Rotenberg, J.-P. Morel, V. Marry, P. Turq, and N. Morel-Desrosiers, Geochim. Cosmochim. Acta 73, 4034 (2009).

${ }^{4}$ R. W. Impey, P. A. Madden, and I. R. McDonald, J. Phys. Chem. 87, 5071 (1983).

${ }^{5}$ G. Hummer, L. R. Pratt, and A. E. Garcia, J. Phys. Chem. 100, 1206 (1996).

${ }^{6}$ S. Koneshan, J. C. Rasaiah, R. M. Lynden-Bell, and S. H. Lee, J. Phys. Chem. B 102, 4193 (1998).

${ }^{7}$ L. X. Dang, J. E. Rice, J. Caldwell, and P. A. Kollman, J. Am. Chem. Soc. 113, 2481 (1991).

${ }^{8}$ L. X. Dang, J. Chem. Phys. 96, 6970 (1992).

${ }^{9}$ S. Koneshan, J. C. Rasaiah, and L. X. Dang, J. Chem. Phys. 114, 7544 (2001)

${ }^{10}$ M. Wilson, P. A. Madden, and B. J. CostaCabral, J. Phys. Chem. 100, 1227 (1996).

${ }^{11}$ R. Vuilleumier and M. Sprik, J. Chem. Phys. 115, 3454 (2001).

${ }^{12}$ T.-M. Chang and L. X. Dang, Chem. Rev. 106, 1305 (2006). 
${ }^{13}$ P. Jungwirth and D. J. Tobias, Chem. Rev. 106, 1259 (2006)

${ }^{14}$ C. D. Wick, I.-F. W. Kuo, C. J. Mundy, and L. X. Dang, J. Chem. Theory Comput. 3, 2002 (2007)

${ }^{15}$ E. Guardia, I. Skarmoutsos, and M. Masia, J. Chem. Theory Comput. 5, 1449 (2009).

${ }^{16}$ H. Yu, T. W. Whitfield, E. Harder, G. Lamoureux, I. Vorobyov, V. M. Anisimov, A. D. MacKerell, Jr., and B. Roux, J. Chem. Theory Comput. 6, 774 (2010).

${ }^{17}$ P. A. Madden and M. Wilson, Chem. Soc. Rev. 25, 339 (1996).

${ }^{18}$ V. Dimitrov and S. Sakka, J. Appl. Phys. 79, 1736 (1996).

${ }^{19}$ R. J. Heaton, P. A. Madden, S. J. Clark, and S. Jahn, J. Chem. Phys. 125, 144104 (2006).

${ }^{20}$ M. Salanne, R. Vuilleumier, P. A. Madden, C. Simon, P. Turq, and B. Guillot, J. Phys.: Condens. Matter 20, 494207 (2008).

${ }^{21}$ B. Rotenberg, M. Salanne, C. Simon, and R. Vuilleumier, Phys. Rev. Lett. 104, 138301 (2010).

${ }^{22}$ N. Marzari and D. Vanderbilt, Phys. Rev. B 56, 12847 (1997).

${ }^{23}$ R. King-Smith and D. Vanderbilt, Phys. Rev. B 47, 1651 (1993).

${ }^{24}$ D. Vanderbilt and R. King-Smith, Phys. Rev. B 48, 4442 (1993).

${ }^{25}$ I. Souza, T. Wilkens, and R. M. Marten, Phys. Rev. B 62, 1666 (2000).

${ }^{26}$ P. L. Silvestrelli and M. Parrinello, Phys. Rev. Lett. 82, 3308 (1999).

${ }^{27}$ L. Bernasconi, M. Wilson, and P. A. Madden, Comp. Mater. Sci. 22, 94 (2001).

${ }^{28}$ L. Bernasconi, P. A. Madden, and M. Wilson, Phys. Chem. Comm. 5, 1 (2002).

${ }^{29}$ A. Aguado and P. A. Madden, J. Chem. Phys. 119, 7471 (2003).

${ }^{30}$ T. Laino and J. Hutter, J. Chem. Phys. 129, 074102 (2008).

${ }^{31}$ P. Umari and A. Pasquarello, Phys. Rev. Lett. 89, 157602 (2002).

${ }^{32}$ P. Umari and A. Pasquarello, Int. J. Quant. Chem. 101, 666 (2005).

${ }^{33}$ R. Resta, Phys. Rev. Lett. 80, 1800 (1998).

${ }^{34}$ The CPMD consortium, CPMD version 3.13.2, URL http://www.cpmd.org.

${ }^{35}$ J. P. Perdew, K. Burke, and M. Ernzerhof, Phys. Rev. Lett. 77, 3865 (1996).

${ }^{36}$ A. Becke, Phys. Rev. A 38, 3098 (1988).

${ }^{37}$ C. Lee, W. Yang, and R. Parr, Phys. Rev. B 37, 785 (1988).

${ }^{38}$ N. Troullier and J. Martins, Phys. Rev. B 43, 001993 (1991)

${ }^{39}$ S. Goedecker, M. Teter, and J. Hutter, Phys. Rev. B 54, 1703 (1996).

${ }^{40}$ C. Hartwigsen, S. Goedecker, and J. Hutter, Phys. Rev. B 58, 1703 (1998).

${ }^{41}$ M. Krack, Theor. Chem. Acc. 114, 145 (2005).

${ }^{42}$ J. Hammond, N. Govind, K. Kowalski, J. Autschbach, and S. Xantheas, J. Chem. Phys. 131, 214103 (2009).

${ }^{43}$ L. X. Dang and B. C. Garrett, J. Chem. Phys. 99, 2972 (1993).

${ }^{44}$ L. X. Dang and P. A. Kollman, J. Phys. Chem. 99, 55 (1995).

${ }^{45}$ H. Berendsen, J. Grigera, and T. Straatsma, J. Phys. Chem. 91, 6269 (1987).

${ }^{46}$ L. X. Dang and T.-M. Chang, J. Chem. Phys. 106, 8149 (1997).

${ }^{47}$ J. VandeVondele, M. Krack, F. Mohamed, M. Parrinello, T. Chassaing, and J. Hutter, Comput. Phys. Commun. 167, 103 (2005).

${ }^{48} \mathrm{CP} 2 \mathrm{~K}$ developers group, URL http://cp2k.berlios.de.

${ }^{49}$ J. VandeVondele and J. Hutter, J. Chem. Phys. 127, 114105 (2007).

${ }^{50}$ R. Car and M. Parrinello, Phys. Rev. Lett. 55, 2471 (1985).

${ }^{51}$ P. Salvador, J. E. Curtis, D. J. Tobias, and P. Jungwirth, Phys. Chem. Chem. Phys. 5, 3752 (2003).
${ }^{52}$ P. Jungwirth, J. E. Curtis, and D. J. Tobias, Chem. Phys. Lett. 367, 704 (2003).

${ }^{53}$ P. Jungwirth and D. J. Tobias, J. Phys. Chem. B 106, 6361 (2002).

${ }^{54}$ P. L. Silvestrelli and M. Parrinello, J. Chem. Phys. 111, 3572 (1999).

${ }^{55}$ C. Sagui, P. Pomorski, T. A. Darden, and C. Roland, J. Chem. Phys. 120, 4530 (2004)

${ }^{56}$ G. D. Mahan, Phys. Rev. A 22, 1780 (1980).

${ }^{57}$ C. Hättig and B. A. Hess, J. Chem. Phys. 108, 3863 (1998).

${ }^{58}$ J. N. Wilson and R. M. Curtis, J. Phys. Chem. 74, 187 (1970).

${ }^{59}$ M. Wilson, P. A. Madden, P. Jemmer, P. W. Fowler, A. Batana, J. Bruno, R. W. Munn, and M. C. Monard, Mol. Phys. 96, 1457 (1999).

${ }^{60}$ P. Jemmer, P. Fowler, M. Wilson, and P. A. Madden, J. Phys. Chem. A 102, 8377 (1998).

${ }^{61}$ G. Lamoureux and B. Roux, J. Phys. Chem. B 110, 3308 (2006).

${ }^{62}$ P. Ren and J. W. Ponder, J. Phys. Chem. B 107, 5933 (2003).

${ }^{63}$ A. Grossfield, P. Ren, and J. W. Ponder, J. Am. Chem. Soc. 125, 15671 (2003).

${ }^{64}$ D. Jiao, C. King, A. Grossfield, T. A. Darden, and P. Ren, J. Phys. Chem. B 110, 18553 (2006).

${ }^{65}$ J.-P. Piquemal, L. Perera, G. A. Cisneros, P. Ren, L. G. Pedersen, and T. A. Darden, J. Chem. Phys. 125, 054511 (2006).

${ }^{66} \mathrm{~J}$. W. Ponder, TINKER Molecular Modeling Package, URL http://dasher. wustl.edu/tinker.

${ }^{67}$ T.-M. Chang and L. X. Dang, J. Phys. Chem. A 113, 2127 (2009).

${ }^{68}$ B. A. Bauer, T. R. Lucas, A. Krishtal, C. V. Alsenoy, and S. Patel, J. Phys. Chem. A 114, 8984 (2010).

${ }^{69}$ P. Bultinck, C. Van Alsenoy, P. W. Ayers, and R. Carbó-Dorca, J. Chem. Phys. 126, 144111 (2007).

${ }^{70}$ R. Vuilleumier and D. Borgis, J. Chem. Phys. 111, 4251 (1999).

${ }^{71}$ D. Marx, M. E. Tuckerman, J. Hutter, and M. Parrinello, Nature 397, 601 (1999).

${ }^{72}$ D. Marx, Chem. Phys. Chem. 7, 1848 (2006).

${ }^{73}$ M. E. Tuckerman, D. Marx, and M. Parrinello, Nature 417, 925 (2002).

${ }^{74}$ M. E. Tuckerman, A. Chandra, and D. Marx, Acc. Chem. Res. 39, 151 (2006).

${ }^{75}$ S. T. Roberts, P. B. Petersen, K. Ramasesha, A. Tokmakoff, I. S. Ufimtsev, and T. J. Martinez, Proc. Natl. Acad. Sci. U.S.A. 106, 15154 (2009).

${ }^{76}$ D. Marx, A. Chandra, and M. E. Tuckerman, Chem. Rev. 110, 2174 (2010).

${ }^{77}$ R. Vácha, V. Buch, A. Milet, J. P. Devlin, and P. Jungwirth, Phys. Chem. Chem. Phys. 9, 4736 (2007).

${ }^{78}$ V. Buch, A. Milet, R. Vacha, P. Jungwirth, and J. P. Devlin, Proc. Natl. Acad. Sci. U.S.A. 104, 7342 (2007).

${ }^{79}$ A. Buin and R. Iftimie, J. Chem. Phys. 131, 234507 (2009).

${ }^{80}$ R. Vácha, T. Megyes, I. Bakó, L. Pusztai, and P. Jungwirth, J. Phys. Chem. A 113, 4022 (2009).

${ }^{81}$ M. Bühl, R. Diss, and G. Wipff, J. Am. Chem. Soc. 127, 13506 (2005).

${ }^{82}$ C. Den Auwer, R. Drot, E. Simoni, S. D. Conradson, M. Gailhanou, and J. Mustre de Leon, New J. Chem. 27, 648 (2003).

${ }^{83}$ R. J. Heaton, R. Brookes, P. A. Madden, M. Salanne, C. Simon, and P. Turq, J. Phys. Chem. B 110, 11454 (2006). 